\title{
HELICAL CHANNELS FOR LONGITUDINAL COMPRESSION OF MUON BEAMS*
}

\author{
G. Penn, J.S. Wurtele, UC Berkeley / LBNL Center for Beam Physics, Berkeley, CA 94720, USA \\ W.M. Fawley, LBNL Center for Beam Physics, Berkeley, CA 94720, USA
}

\begin{abstract}
Transverse cooling of muon beams can be achieved through ionization cooling. However, longitudinal emittance growth occurs as a by-product of this method; at the muon energy of choice, low energy muons suffer enhanced energy loss. Even a small amount of longitudinal cooling would be advantageous for a neutrino factory, while longitudinal cooling by a factor of 100 is required for a muon collider. We consider 6D cooling using a helical wiggler channel inside a uniform solenoid field, which results in radial dispersion, which is combined with wedge-shaped absorbers to correct the energy spread and transfer longitudinal emittance into the transverse degrees of freedom. These systems are challenging because of the large dispersions required, and because the muon beams have large energy spread and non-zero transverse emittance. We describe scenarios which, in simulations, yield significant reduction of energy spread for unbunched beams. We explore the demands and performance of such channels, as well as the feasibility of longitudinal compression for bunched beams.
\end{abstract}

\section{INTRODUCTION}

The method of choice for achieving cooling of muon beams is ionization cooling [1], in order to achieve cooling time scales shorter than the muon lifetime of $\approx 2$ $\mu \mathrm{s}$. However, ionization cooling typically only cools the beam in the transverse directions and, for typical parameters, causes longitudinal heating because of the variation in energy loss particles experience at different energies. To first order, the longitudinal emittance growth is given by $\epsilon_{L}^{\prime} \simeq \epsilon_{L}(d / d E)(d E / d s)$. Thus, some form of longitudinal cooling is essential for a muon collider [2] and may be an important part of a neutrino factory [3].

This process is known as emittance exchange because such longitudinal cooling is done at the expense of either increased transverse emittance, or reduced transverse cooling rates. Most schemes currently being considered for emittance exchange rely on placing wedge-shaped absorbers in regions of high dispersion. The systems being considered include bent solenoids, bent lithium lenses, ring coolers, and helical wigglers. Here, helical wigglers are described and examples to reduce the energy spread of a muon beam are given. Alternative methods to perform transverse cooling include stopping a beam, followed by rapid reacceleration; and rotating the matched phase space

\footnotetext{
* Work supported by the U.S. Department of Energy, grant DEFG-0395ER-40936.
}

distribution out of the transverse plane, so that all components of motion experience the ionization cooling. Two examples are given which reduce the energy spread of a beam, and which correspond to beam parameters at different stages in the front end of the BNL Feasibility Study $2[4,5]$ design. The first reduces the longitudinal emittance of a bunched beam similar to that at the end of the cooling channel, the second example reduces the energy spread of the beam after the first induction linac of the phase rotation section.

\section{HELICAL WIGGLER PROPERTIES}

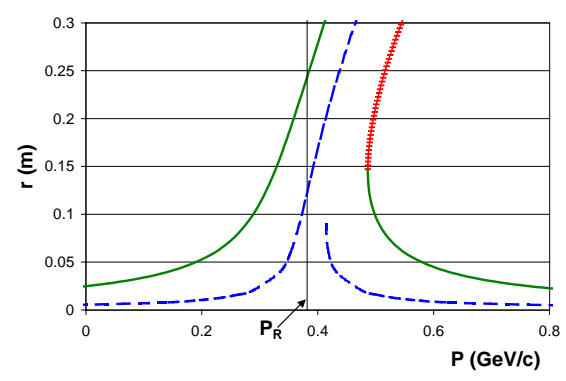

Figure 1: Radius of reference orbit as a function of momentum. Red, hatched line corresponds to unstable orbits. Blue, dashed line corresponds to stable orbits when dipole field is reduced by factor of 5 . Resonant value of momentum is indicated.

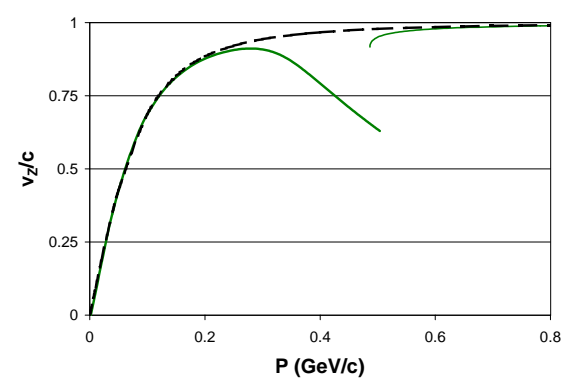

Figure 2: Forward velocity of reference orbit as a function of momentum. Velocity for motion in straight line (dashed line) is shown for comparison. 
A helical wiggler consists of a uniform solenoid field and a rotating dipole field. The magnetic fields are given by

$$
\begin{aligned}
& B_{x}=-2 B_{W} I_{1}^{\prime}\left(\kappa_{W} r\right) \sin \left(\kappa_{W} z\right), \\
& B_{y}=2 B_{W}\left[I_{1}\left(\kappa_{W} r\right) / \kappa_{W} r\right] \cos \left(\kappa_{W} z\right), \\
& B_{z}=B_{0}-\kappa_{W}\left(x B_{y}-y B_{x}\right),
\end{aligned}
$$

where $I_{1}$ is the modified Bessel function of first order, and $\kappa_{W}=2 \pi / L_{W}$, where $L_{W}$ is the period of rotation of the dipole field.

The stable orbit radius varies with energy, with transverse velocity that scales as $v_{\perp} \propto B_{W} / E$, and $r \propto$ $B_{W} /\left(\kappa_{W} E\right)$. There is a resonance when the cyclotron motion has the same period as the dipole rotation, when $P_{R}=e B_{0} L_{W} / 2 \pi$; the dispersion increases as the momentum approaches the resonant value. The reference orbit radius and forward velocity are shown in Figures 1 and 2 for the channel used in the cooled beam example. The wiggler parameters chosen are $L_{W}=2.5 \mathrm{~m}, B_{0}=3.2 \mathrm{~T}$, $B_{W}=0.2 \mathrm{~T}$.

\section{RESULTS}

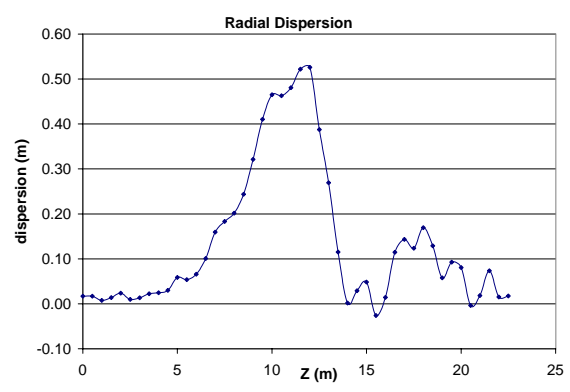

Figure 3: Energy dispersion carried by beam in helical wiggler emittance exchange channel.

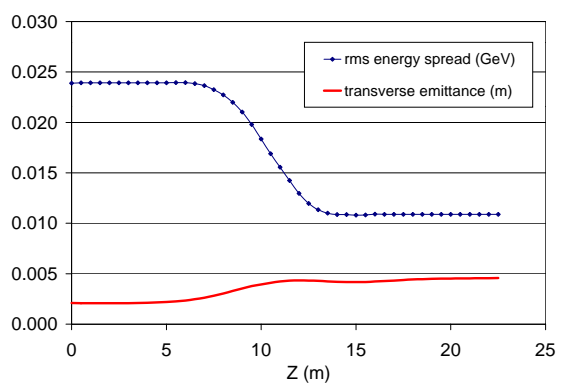

Figure 4: Variation in energy spread and transverse emittance in helical wiggler emittance exchange channel. Solenoid field is a uniform 3.2 $\mathrm{T}$ throughout.

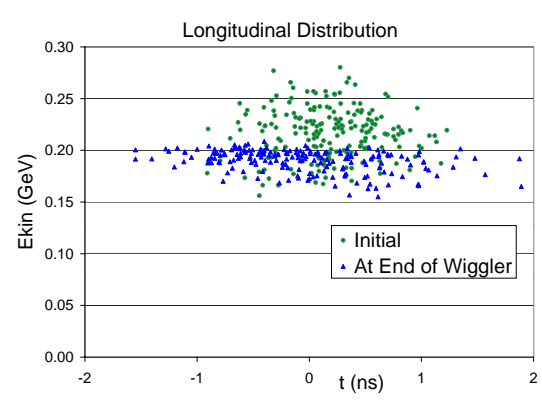

Figure 5: Initial longitudinal phase space distribution of beam compared against final distribution.

To accomplish longitudinal cooling, wedges of beryllium are place in regions of large beam dispersion, reducing the energy spread at the expense of increasing transverse emittance. The results of the channel, simulated in ICOOL [6], are shown in Figures $3-5$. The momentum of the beam is $300 \mathrm{MeV} / \mathrm{c}$; the other beam parameters are comparable to those at the end of the Study 2 cooling channel, with longitudinal emittance $\approx 28 \mathrm{~mm}$, and transverse emittance $\approx 2 \mathrm{~mm}$. The channel, including a ramp in and out of a $3.2 \mathrm{~T}$ solenoid section, is $23 \mathrm{~m}$ long, and 21 beryllium wedges are placed between $z=6$ and $16 \mathrm{~m}$. The energy spread of the beam is reduced by a factor of 2 . The beam remains bunched without the presence of rf cavities because the channel is isochronous, and all particles have forward velocities close to the maximum allowed by the channel for momenta below resonance. However, the beam is no longer matched to the original rf system.

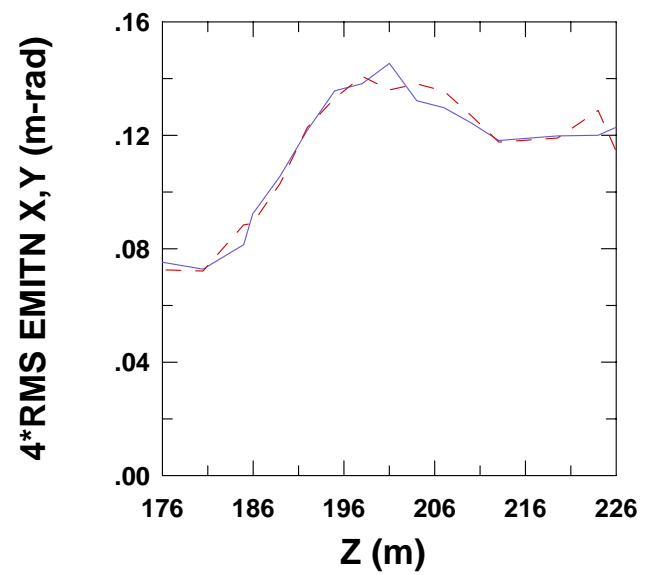

Figure 6: Transverse emittances of beam.

The second emittance exchange example reduces the energy spread of the beam at the end of the first induction linac in the BNL Feasibility Study 2 front-end. Here, the solenoid field ramps up from 1.25 to $3 \mathrm{~T}$, and the wiggler period is $L_{W}=6 \mathrm{~m}$. The dipole field is gradually increased to $0.085 \mathrm{~T}$. The full channel is $48 \mathrm{~m}$ long, with 30 

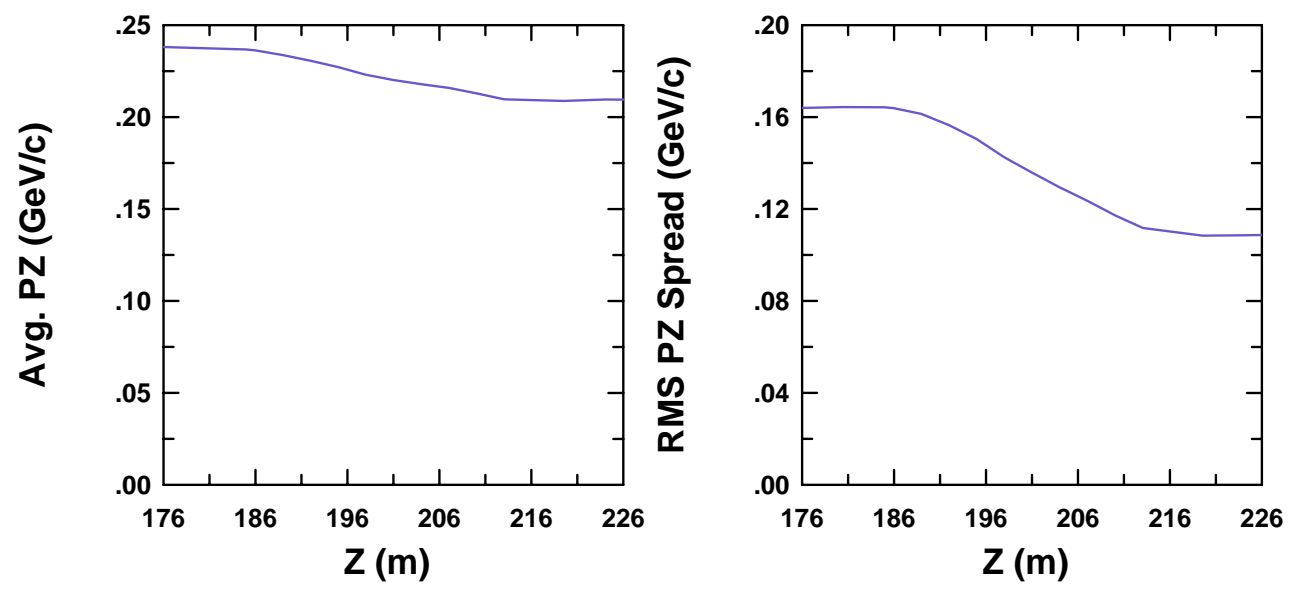

Figure 7: Average momentum and momentum spread of beam.

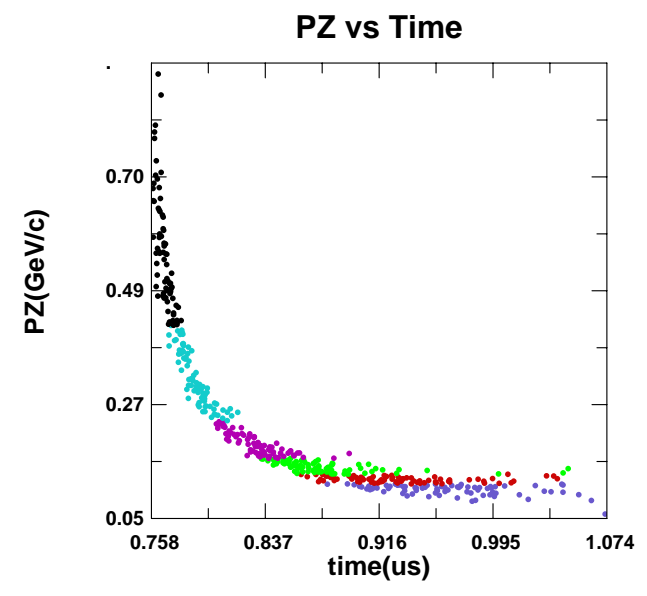

Figure 8: Initial longitudinal phase space distribution of beam, color-coded for initial particle momentum.

liquid hydrogen annular absorbers each $1 \mathrm{~m}$ thick. Low energy particles pass below the minimum radius of the absorber, while high energy particles pass through the absorbers and have their energy reduced.

The results of this channel are shown in Figures 6-9. The total spread of energies is reduced; however, the energy density within the capture region of the downstream channel is not improved, as the high energy particles are brought down in energy to just above this range. Implementation of such a lattice may require re-optimization of the waveforms or length of the downstream induction linacs.

These preliminary results show the promise of helical wiggler channels for reduction of longitudinal emittance; however, examples have not yet been determined which are directly applicable to the neutrino factory design without modifications.

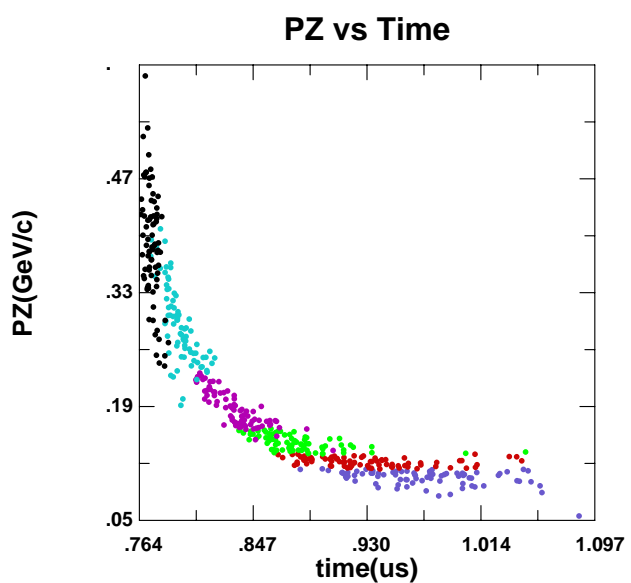

Figure 9: Longitudinal phase space distribution of beam after helical wiggler section, color-coded for initial particle momentum.

\section{REFERENCES}

[1] A.N. Skrinsky and V.V. Parkhomchuk, Sov. J. Part. Nucl. 12, 223 (1981).

[2] C.M. Ankenbrandt et al. (Muon Collider Collaboration), Phys. Rev. ST Accel. Beams 2, 081001 (1999).

[3] S. Geer, Phys. Rev. D57, 6989 (1998).

[4] "Feasibility Study-II of a Muon-Based Neutrino Factory" BNL-52623, eds. S. Ozaki, R. Palmer, M. Zisman, J. Gallardo.

[5] R. Fernow, J. Gallardo, R. Palmer and P. Lebrun, "Ionization Cooling Scenario for a Neutrino Factory", paper FPAH079, PAC'01, Chicago, June 2001.

[6] R.C. Fernow, in Proceedings of the 1999 Particle Accelerator Conference, NY, p.3020. 